\title{
Spanish Speakers with Neurological and Psychiatric Disabilities: Relevant Factors Related to Rehabilitation
}

The number of people with neurological and psychiatric disabilities in Spanish-speaking countries has been increasing over the past two decades [1]. Due to the many physical, cognitive, behavioral, and emotional difficulties that individuals with disabilities and their families face, there is a great need for rehabilitative services. However, Spanish-speaking individuals, both in the US and abroad, have less access to these services. In Spanish-speaking developing countries especially, support groups, individual psychological treatment, vocational and financial assistance, and caregiver resources are scarce [2]. This special issue presents eight studies that collectively assess important factors that should be considered in the rehabilitation of Spanish-speaking individuals with neurological and psychiatric disabilities, such as brain injury and Schizophrenia.

One important step toward developing rehabilitation programs for Spanish-speaking individuals with traumatic brain injury (TBI) is to investigate factors associated with the short- and long-term psychosocial consequences of injury. Though much work has been conducted in this area for Anglo-Saxon populations, very little work has focused on Spanish-speaking individuals. As cultural differences may influence the rehabilitation process, this is an important area of focus [2,3]. In this special issue, two studies examine the predictors of satisfaction with life and employment, which are considered to be two of the most important outcomes associated with successful rehabilitation for individuals with TBI.

Ketchum and colleagues explore which outcomes are associated with competitive employment one year postinjury in Hispanic patients with TBI. Of the 418 patients from the TBI Model Systems (TBIMS), 72.2\% were not competitively employed at one year post-injury, as measured by the dichotomous variable of "competitively employed" or "not competitively employed." Further, pre-injury employment status, pre-injury level of education, etiology, associated SCI, PTA, DRS at discharge, FIM cognitive, FIM motor, and LOS in acute and rehabilitation care were all associated with employment status one year post-injury. These outcomes need to be considered when Hispanic patients wish to obtain competitive employment post-injury.

Also, Ketchum and colleagues aim to determine the demographic, injury, rehabilitation, and followup characteristics associated with satisfaction with life (SWL) in Hispanic patients with TBI at one year postinjury. Retrospective analysis of 291 Hispanic patients with TBI from the TBI Model Systems (TBIMS) showed that at one year post-injury, age at injury, associated spinal cord injury (SCI), employment status post-injury, DRS post-injury, FIM cognitive, and FIM motor were associated with SWL, as measured by the satisfaction with life scale (SWLS). This implies that rehabilitation efforts should focus on cognition and vocational skills to improve SWL for Hispanic patients with TBI.

Cognitive rehabilitation is an important form of treatment to manage the cognitive problems associated with neurological and psychiatric disability [4]. However, the majority of cognitive rehabilitation interventions are in English and very few programs exist for Spanish speakers. It is important to study the applicability of established cognitive rehabilitation interventions on Spanish-speaking individuals. This special issue presents four studies that examine the applicability, efficacy, and effectiveness of different cognitive rehabilitation programs and techniques in Spanish-speaking populations. 
In their meta-analysis of 24 studies, Guardia and colleagues assess the effects of neuropsychological rehabilitation on the quality of life (QOL) of Spanishspeaking individuals with TBI. They also explore other factors that mediate this relationship. It was found that QOL can improve in certain conditions with neuropsychological rehabilitation, but this change is not permanent.

In a study exploring cognitive deficits that affect patients with chronic Schizophrenia, Ojeda and colleagues go beyond typical pharmacological treatments and explore the efficacy of neuropsychological rehabilitation for Spanish-speaking patients with chronic Schizophrenia. They recruited 67 patients in a long-stage united for Treatment Resistant Patients with Schizophrenia (UPR). Patients were placed in a cognitive rehabilitation group (Rehacop) or Occupational Therapy (OT) group, which added either neuropsychological or occupational rehabilitation to their preexisting standard treatments. At post-treatment, the cognitive rehabilitation group showed significant clinical and cognitive improvement over the OT group. This shows that adding neuropsychological rehabilitation to preexisting treatment significantly helps patients with chronic Schizophrenia.

In another study, de los Reyes and colleagues examine the moderating effect of cognitive impairment (CI) on the usefulness of the generation effect to improve learning and memory in Spanish-speaking individuals with TBI. Sixty-one individuals with TBI (29 without CI, 22 with mild to moderate CI, and 10 with severe $\mathrm{CI}$ ) and 44 healthy controls (HC) were required to remember the last word in each of 32 sentences. Target words were presented in a self-generated and provided condition. Recall and recognition were examined immediately, after 30 minutes, and at one week. Individuals remembered and recalled significantly more words in the generated condition than the provided condition, regardless of group or time. The self-generation technique equally benefitted all participants regardless of TBI status or degree of CI.

Further, Caracuel and colleagues explore the longterm effectiveness of a holistic neuropsychological rehabilitation program (HNRP) for Spanish-speaking patients with acquired brain injury (ABI). They also assess whether there is an outcome difference between patients with a time since injury of less than six months or greater than six months. Eighteen patients and their relatives were part of the HNRP for six months. At the conclusion of the HNRP, patients with the greater time since injury showed improved cognitive and executive functioning, social and emotional self-regulation, apathy, and mood. It was also found that patients with less time since injury showed more improvement in mood and cognitive functioning than the patients with a greater time since injury. Thus, the HNRP is an effective program for mood and cognitive functioning in patients with ABI.

The physical, emotional, social, and behavioral symptoms of TBI present significant challenges for not only survivors, but also their loved ones [5,6]. Rather than considering family members as merely support systems for people with TBI, the majority of researchers and clinicians recognize that the entire family system, as well as the individuals within it, simultaneously influence and are influenced by one another and the injury [5]. However, two major limitations exist in the literature on this topic. First, most of what is known about the experience of family members of individuals with TBI is based on research with Anglo-Saxon individuals. As a result, little is known about family members of individuals with TBI in Spanish-speaking countries. Second, the majority of research in this area includes only one member of a family. Consequently, there is limited information about the bidirectional influence of family members in the face of TBI. Two articles in this special issue represent attempts to fill these gaps in the literature.

Lehan and colleagues examined 51 pairs of individuals with TBI and their caregivers in Colombia. After controlling for survivor education and history of occupational therapy, they found that survivors receiving care from a family member who reported a higher level of burden had poorer objective neuropsychological functioning than those receiving care from a family member who reported a lower level of burden.

Lehan and colleagues described the levels of family adaptability, cohesion, communication, and satisfaction in 38 pairs of individuals with TBI and their caregivers in Mexico. They also tested one hypothesis of the Circumplex Model. Finally, the authors explored the influence of perceptions of family adaptability as reported by the individuals with TBI and their caregivers on their own as well as the other's reports of family communication and satisfaction. They found that most individuals with TBI and their caregivers reported balanced family adaptability and cohesion and relatively high levels of family communication and satisfaction. In addition, they reported that TBI survivors and family caregivers who reported greater levels of family adaptability and cohesion also endorsed better family communication and greater family satisfaction. 
In addition, individuals with TBI whose family caregiver endorsed balanced family adaptability and cohesion reported better family communication. Further, family caregivers of TBI survivors who reported balanced family adaptability and cohesion reported better family communication.

The articles in this special issue assess important factors that should be considered in the rehabilitation of Spanish-speaking individuals with neurological and psychiatric disabilities and are among the first in the literature to address the unique characteristics of these individuals. Collectively, the findings provide guidance to clinicians and highlight the need for further research in this area.

Guest Editor

Juan Carlos Arango-Lasprilla, Ph.D. Associate Professor, Department of Physical Medicine and Rehabilitation,

Virginia Commonwealth University, Richmond, VA,

USA

Email: jcarangolasp@vcu.edu

Tel.: +1 8048288797

Fax: +1 8048282378

\section{References}

[1] Razzouk, D., Zorzetto, R., Dubugras, M.T., Gerolin, J., \& de Jesus Mari, J. (2007). Leading countries in mental health research in Latin America and the Caribbean. Revista Brasileira de Psiquiatria, 29, 118-122.

[2] Arango. J.C., \& Kreutzer, J. (2010). Racial and ethnic disparities in functional, psychosocial, and neurobehavioral outcomes after brain injury. Journal of Head Trauma Rehabilitation, 25, 128-136.

[3] Niemeier, J., \& Arango J.C. (2007). Toward improved provider practice competency in rehabilitation services for ethnically diverse survivor's of Traumatic Brain Injury. Journal of Head Trauma Rehabilitation, 22, 75-84.

[4] Wilson, B.A. (1997). Cognitive rehabilitation: How it is and how it might be. Journal of the International Neuropsychological Society, 3, 487-496.

[5] Godwin, E., Kreutzer, J., Arango, J.C., \& Lehan, T. (2011). Marriage after brain injury: Review, analysis and research recommendations. Journal of Head Trauma Rehabilitation 26, 43-55.

[6] Kreutzer, J., Marwitz, J., Godwin, E., \& Arango, J.C. (2010). Practical approaches to effective family intervention after brain injury. Journal of Head Trauma Rehabilitation, 25, 113120. 